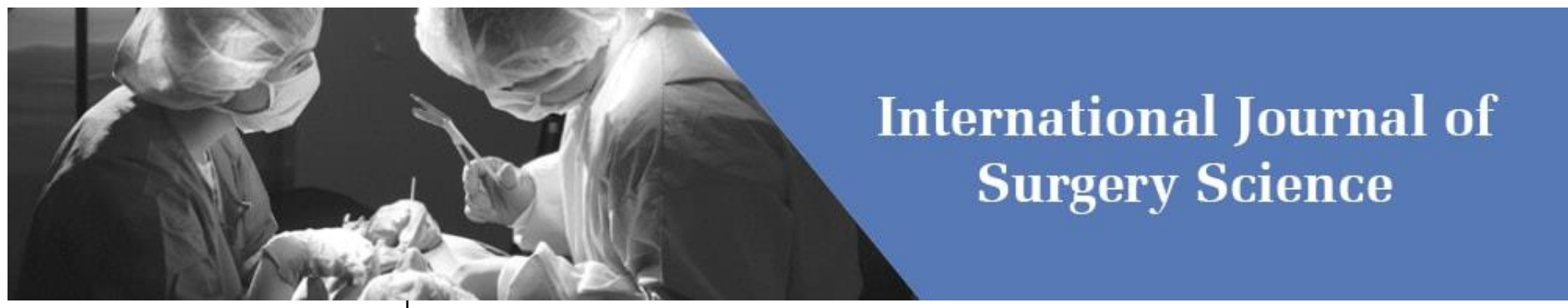

E-ISSN: 2616-3470

P-ISSN: 2616-3462

(C) Surgery Science

www.surgeryscience.com

2021; 5(2): 132-134

Received: 12-02-2021

Accepted: 17-03-2021

Dr. KM Ganesh Babu

Associate Professor in General

Surgery, Akash Institute of

Medical Sciences, AIMS \& RH and

Research Hospital, Devanahalli,

Bangalore Rural, Karnataka, India

Dr. BR Venkatkrishna

HOD General Surgery, AIMS \&

RC, Dr. Balakrishna. S.V,

Professor in General Surgery,

AIMS \& RH Devanahalli,

Bangalore Rural, Karnataka, India
Corresponding Author:

Dr. Elizabeth Anna Samue

Post Graduate Student,

Department of ENT, Sree

Mookambika Institute of Medical

Sciences, Kulasekharam,

Tamil Nadu, India

\section{Evaluation of Alcohol in Pancreatitis}

\section{Dr. KM Ganesh Babu and Dr. BR Venkatkrishna}

\section{DOI: https://doi.org/10.33545/surgery.2021.v5.i2c.673}

\section{Abstract}

The aim of this retrospective study is to identify, causes of Acute Pancreatitis in rural population and the conservative treatment method practiced in Akash Medical College \& RH. Devanahalli.

Methodology: About 100 patients were selected, diagnosed as Acute Pancreatitis using biochemical and Radiological investigations, this retrospective study was done for a period of 2 years

Results: In this study the major risk factor associated with Acute Pancreatitis was chronic alcohol intake, majority were male patients $(98 \%)$ and purely medical treatment was done.

Conclusion: The most common cause of Pancreatitis in this rural place is chronic alcohol intake with mild to moderate severity. Treatment followed was only medical with no surgical intervention.

Keywords: Acute pancreatitis, Alcohol, severity, medical

\section{Introduction}

Acute pancreatitis is defined as in flammation of the pancreas and restitution of the gland if the primary cause is eliminated. Progressive inflammation may lead to haemorrhagic and necrotic pancreatitis.

\section{Etiology of Pancreatiti}

Metabolic: Alcohol, hyperlipoproteinemia, hypercalcaemia, Drugs, Scorpion venom

Mechanical: Cholelithiasis, Post-operative (Gastric or Biliary Surgery), Post traumatic, Duct obstruction, Pancreatic tumour, duodenal obstruction.

Vascular-Post operative (cardiac pulmonary by pass), Polyarteris nodosa, Athero embolism

Infection-Mumps, coxsackie virus infection ERCP, Trauma

Idiopathic: (especially in India) ${ }^{[1]}$.

Development of Pancreatitis: Etiological factors mentioned above initiate 'bile reflux' leading to acute pancreatitis, manifested as edema, vascular injury and pancreatic acinar damage Inflammation causes activation of pancreatic enzymes.

Trypsin/phospholipase A etc. leading to auto digestion of pancreas and necrosis. Ethanol \& Acetaldehyde activate pancreatic stellar cells via Kinase pathways ${ }^{[1]}$.

Pathogenesis of alcohol injury induced acute pancreatitis:

- Alcohol Stimulates gastric acid secretion and the duodenal acidification releases 'secretin', which causes increased secretion of water and bicarbonate.

- 2.Alcohol induces 'spasm' of the sphincter of oddi causing partial obstruction to pancreatic secretion flow, which further increases exocrine secretion of pancreas and distension of the ductal system with raised tension.

- Alcohol induces raised tension in the ductal system of Pancreas, increased intraductal pressure increases Permeability of the pancreatic ducts to macromolecules

- All the above mentioned pathophysiology causes extravasation of enzymes causing pancreatic injury due to presence of proteins.

- Alcohol injury causes hypertriglyceridemia with raised Levels of free fatty acids, causing acinar cell injury and damage to capillary endothelium Pancreas pathologically becomes edematous, Intralobular septa become infiltrated with inflammatory Cells. 
Microscopically gland shows fat necrosis, extending fat necrosis to surrounding tissues with Calcium deposition causes' white yellow plaque deposition'.

Vascular thrombosis with disruption also contributes to necrosis and haemorrhagic infarction Enzyme levels are elevated due to release from pancreas Leading to peritoneal exudates and blood Other systems affected in Pancreatitis are

- fluid \& electrolyte changes.

- Cardiovascular changes

- Respiratory changes

- Renal changes

- Local changes

Fluid \& electrolyte imbalance causes decrease in circulatory blood volume due to loss in retroperitoneum,

Hypocalcaemia \& hypomagnesemia accompanies it.

The above leads to hypotension, tachycardia, circulating vasoactive substances and increased peripheral vascular resistance leads to decreased cardiac output Arterial hypoxemia, decreased pulmonary compliance, Gas diffusion capacity decreases across the alveolar membrane, pleural effusion, decrease in lecithin of pulmonary surfactant due to pancreatic lecithinase, free fatty acids and proteolytic cleavage complements are the alterations in the Pulmonary system.

The major cause of death in Pancreatitis is because of Renal failure (hypovolemia \& deposition of fibrin complexes in glomeruli) Other systemic features are raised serum bilirubin, elevated Alkaline phosphatase, decreased platelet count \& fibrinogen due to raised Pancreatic Proteolytic enzymes.

Local manifestations are Paralytic ileus, Ascites, Duodenal and biliary obstruction, peri pancreatic tissue destruction, persistent chronic pseudocyst in $1 \%$ patients ${ }^{[2]}$, Pancreatic abscess formation due to secondary infection in 1-9\% patients, colonic perforation in $1 \%$ patients especially left side transverse colon/splenic flexure. Inflammatory process \& formation of pseudocysts can obstruct the pancreatic, bile ducts \& duodenum [2].

\section{Clinical features of Acute pancreatitis:}

Abdominal pain (90-100\%) Nausea and Vomitting (70-80\%)

Shock (10\%), hypotension, sweating, dry tongue were the clinical features seen in our hospital.

Physical Examination-restless patient with a forward bent attitude, tachycardia, tacypnoea and hypotension Abdominal distension, epigastric fullness Tenderness in epigastrium left hypochondrium [2], grey turners sign, cullens sign may be present Patient can also have left sided pleural effusion, confusion, psychoses due to hypoxemia, cerebral fat embolism or DIC.

Laboratory Investigations: In our study biochemical parameters studied were Serum ${ }^{[3]}$ Amylase, Serum Lipase \& Urine amylase. In our study S. Amylase was elevated in $98 \%$ cases, S. Lipase was elevated in $90 \%$ cases. Hypocalcaemia seen in $60 \%$ cases elevated levels of alkaline phosphatase were seen in $30 \%$ cases, Leucocytoses $-11000-29000$ were seen in $98 \%$ cases raised Haematocrit was seen in $70 \%$ cases ${ }^{[4]}$.

Radiological Investigations-Erect Abdomen Xray showed

'Sentinel loop sign ' $\&$ ' colon cut off sign' in $35 \%$ cases,

Chest $x$-ray showed pleural effusion in $20 \%$ cases Ultrasonography Abdomen showed enlarged \& edematous pancreas, pseudocyst of pancreas $\&$ abscess of the pancreas ${ }^{[5-6]}$. CT Scan-showed enlarged pancreas, obliteration of fat planes, Stranding of fat $\&$ fat thickening \& ascites (85\%), but MRI scan is more superior to CT scan.

Treatment: Given in this study was as follows
Nutrition: patient kept NBM for 3-5 days, as the symtoms

like vomiting, gastritis, abdominal distension decreased and when patient could tolerate orally patient was started with liquids after 4-5 days. But present trend is to start enteral feeding nasogastric or naso jejunal. Studies have shown that enteral nutrition is better than TPN Abdominal distension was measured every hourly. Gastritis and vomiting was treated with RYLES Tube aspiration, inj Pantoprazole IV $40 \mathrm{mg}$ bd or tid based on symptoms of gastritis, Pantoprazole was continued for nearly till time of discharge from hospital In $\mathrm{j}$ Emeset was used as necessary $4^{\text {th }} 6^{\text {th }}$ or $8^{\text {th }}$ hourly.

Fluid Management: Crystalloids and Colloids were used in ratio of 3:1. IV DEXTRAN improves the microcircu lation monitored by haematocrit levels (35-40\%). CVP around 8-12 cm of $\mathrm{H}_{2} \mathrm{O}$.

Pain management: Inj Tramadol, inj paracetamol and Inj meperidine was used, in three cases with severe pain Epidural analgesia was used ${ }^{[8]}$.

Antibiotics used were Imipenem, Meropenem, Metrogyl fluoroquinolones \& Cephalosporins ${ }^{[7-8]}$ trials were conducted previously stressing the importance of Antibiotics ${ }^{[7-8]}$.

Inj Somatostatin $4 \mathrm{mg}$ tid/qid was used and tapered 2 patients were subjected to peritoneal lavage, but patients became bad and referred to higher centre Oxygen inhalation and Ventilatory support was also used accordingly. In cases of pseudocysts of pancreas serial sonographies showed that cysts decreased in size Prognostic indicators used were RANSONS Criteria, BISAP Score, APACHE II SCORE, CRP assays, CT severity [9]. Interventional radiologists have started doing image guided aspirations and debridement of necrotic material

\section{Materials and methods}

Patients were all those admitted to AIMS \& RH and patients are from Devanahalli and surrounding rural areas Inclusion criteria patients belonged to the age group 11-60 yrs, patients with clinical features of acute pancreatitis, raised serum amylase and lipase, CT features of acute pancreatitis.

Exclusion criteria: patients with malignancies, previous abdominal surgeries, children less than $10 \mathrm{yrs}$ and patients with other acute abdomens like hollow viscus perforation and intestinal obstruction, pregnant patients

Criteria studied: severity of acute pancreatitis, pancreatic necroses, response to mode of medical treatment Study period was from March 2018- March 2020 and the sample size was 100 , study design was retrospective observational study

Results-Statistical analyses reported as mean of $+/$ - of standard deviation. In this study of pancreatitis $95 \%$ were mild pancreatitis (interstitial) and 5\% were severe pancreatitis (necrotizing).

\begin{tabular}{|c|c|c|c|}
\hline Age & Male & Female & Total \\
\hline $11-20 \mathrm{yrs}$ & 2 & & 2 \\
\hline $21-30 \mathrm{yrs}$ & 8 & & 8 \\
\hline $31-40 \mathrm{yrs}$ & 22 & 2 & 24 \\
\hline $41-50 \mathrm{yrs}$ & 42 & & 42 \\
\hline $51-60 \mathrm{yrs}$ & 16 & & 16 \\
\hline$>60$ Yrs & 8 & & 8 \\
\hline
\end{tabular}

Maximum no of patients were in age group 41-50 yrs minimum patients were in age 11-20 yrs males, females were 2 in age group 31-40 yrs In our study $95 \%$ had been diagnosed as mild pancreatitis (interstitial pancreatitis) and 5\% severe pancreatitis (Necrotising pancreatitis). 


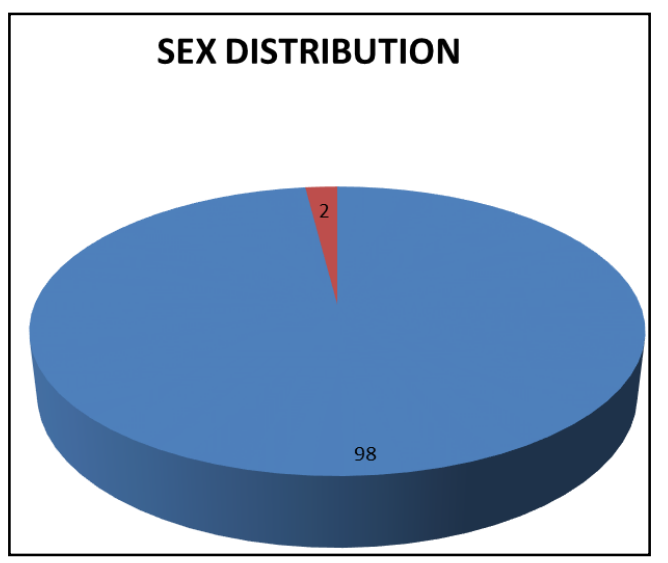

Chart Showing Male Preponderance Males 98\% and females 2\%

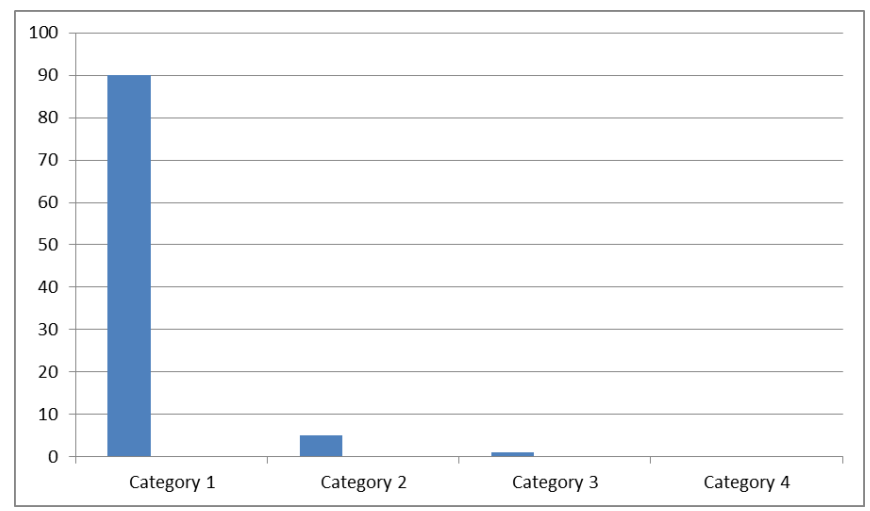

Causes of Pancreatitis in this study-category 1. Alcohol

2. Gall stones +Alcohol 3. Pseudopancreatic Cyst. 4. Other causes

Discussion: In this study of 100 patients. majority were male patients (96), females (2), children (2) Study shows that Pancreatitis is more common in males, maximum incidence is in age 41-50 yrs Commonest cause of pancreatitis is Alcohol,1 person had pseudocyst of pancreas with calculi, severity of pancreatitis is graded as mild, moderate and severe by CT severity index $92 \%$ mild grade, $7 \%$ moderate \& $2 \%$ severe, this study shows most prevalent form of pancreatitis is mild which can be managed conservatively without surgical intervention, 1 male patient aged above 60 yrs was severe and referred. Serum amylase levels reduced in 4 to 6 days but Serum lipase levels were elevated till 8 to 14 days in patients.

Conclusion: Acute Pancreatitis is more prevalent in the male population, more prevalent in age group 41-50 yrs, the leading cause of pancreatitis in this study was chronic alcohol intake, gall stones contributing a small percentage but they were also Alcoholics, where as in urban cities gall stones are the predominant cause of pancreatitis. Serum amylase levels may be normal in some patients, rarely Serum lipase may also be normal, then CT SCAN can help in diagnosing Pancreatitis and CT SCAN is considered the gold standard in diagnosing pancreatitis. In our set up which is having rural population most of the cases can be managed conservatively without surgical intervention

\section{References}

1. Sakorafas G H. Etiology \& Pathogenesis of Acute pancreatitis. j clin gastro 2000;30:343-356.

2. Beger HG et al. Natural course of Acute Pancreatitis; world j surg; 21:130-135.

3. Corsetti j cox.' Combined serum amylase \& lipase determinations for diagnoses of acute pancreatitis' Clin Chem 39(12):2495.9.

4. Forsmark CE, Baillie J. AGA. Institute technical review on Acute Pancreatitis. Gastroenterology 2007;132:2022-2044.

5. RANSON J: diagnostic standards of acute pancreatitis world j surg 1997;21:136.

6. Balthazar E J, Ranson j h. Acute pancreatitis: value of CT in establishing prognosis, Radiology 1990;174(2):331-6.

7. Golub $\mathrm{R}$ et al. Role of antibiotics in acute pancreatitis; a meta analysis. J Gastrointest Surg 1998;2(6):496-503.

8. Niesel HC et al. Epidural blockade for analgesia \& treatment of acute pancreatitis, Reg Anaest 1991;14(6):97100.

9. Losada M, Jorge. Retrospective review of 251 patients with acute pancreatitis; source: Hevistachikna de cirugia 2010 , 62(6):557-563. 